www.jmscr.igmpublication.org

Index Copernicus Value: 79.54

ISSN (e)-2347-176x ISSN (p) 2455-0450

crossref DOI: https://dx.doi.org/10.18535/jmscr/v7i6.157

\title{
Knowledge and awareness of health care providers about health care associated infection control measures in neonatal intensive care units of a tertiary care hospital of Himachal Pradesh
}

\author{
Authors \\ Dr Mohit Karol ${ }^{1}$, Dr Sunidhi Karol ${ }^{2 *}$ \\ ${ }^{1}$ Senior Resident, Department of pediatrics, IGMC, Shimla \\ ${ }^{2}$ Junior resident, Department of community medicine, PGIMS, Rohtak \\ *Corresponding Author \\ Dr Sunidhi Karol
}

Address: Karol Cottage, Near Saw Mills, Lakkar Bazaar, Shimla, Himachal Pradesh, Pin: 171001, India

\begin{abstract}
Background: Health care-associated infection (HCAI), also referred to as "nosocomial" or "hospital" infection, is an infection occurring in a patient during the process of care in a hospital or other health care facility which was not present or incubating at the time of admission.

Material \& Methods: A cross sectional study was carried over a period of two months in NICU settings of IGMC, Shimla. 15 staff nurses and 20 residents (Junior residents \& Senior residents). Thus, sample size of 35 was covered.

Results: All of the staff were adequately qualified for the work in ICU although no. of staff was below standard for an ICU. Most of the staff was found to be aware of HCAI, hand hygiene, personal protection and BMW segregation but at the same time not all were practicing hand hygiene and personal protection all the time during patient care. Some of the staff were also found to be well trained in infection control measures. In fact, few were even not knowing about existence of HICC, ICP and ICG. All the shortcomings in infrastructure and staff increases the risk if HCAI as shown by previous studies.

Conclusion: Hand hygiene is of most important in preventing HCAI. But, unfortunately it is neglected in Indian setting specially in government setup where work load is in excess and lack of materials required for hand hygiene are lacking. Staff should be motivated to follow infection control guidelines, hand hygiene, personal protection, and trained enough, so that, this major problems of HCAI

Keywords: NICU, PICU, HCAI, Infection control, Knowledge, Awareness.
\end{abstract}

\section{Introduction}

Health care-associated infection (HCAI), also referred to as "nosocomial" or "hospital" infection, is an infection occurring in a patient during the process of care in a hospital or other health care facility which was not present or incubating at the time of admission. HCAI can affect patients in any type of setting where they receive care and can also appear after discharge. Furthermore, they include occupational infections among staff. HCAI represents the most frequent adverse event during care delivery and no 
institution or country can claim to have solved the problem yet. Based on data from a number of countries, it can be estimated that each year, hundreds of millions of patients around the world are affected by HCAI. The burden of HCAI is several folds higher in low- and middle-income countries than in high-income ones. ${ }^{1}$

Newborns are at higher risk, with infection rates in developing countries 3-20 times higher than in high-income countries. Among hospital-born babies in developing countries, health careassociated infections are responsible for $4 \%$ to $56 \%$ of all causes of death in the neonatal period, and $75 \%$ in South-East Asia and Sub-Saharan Africa. Surgical site infection is the leading infection in the general patient population in countries with limited resources, affecting up to two third of operated patients and with a frequency up to nine times higher than in developed countries. Every day, HCAI results in prolonged hospital stays, long-term disability, increased resistance of microorganisms to antimicrobials, massive additional costs for health systems, high costs for patients and their family, and unnecessary deaths. Thus, this study is designed to determine the infection control practices among health care professionals in a tertiary care hospital. ${ }^{2}$

\section{Methodology}

A cross sectional study was carried over a period of two months in NICU settings of IGMC, Shimla. This is 872 bedded tertiary care government hospital of Himachal Pradesh. The hospital is also a referral Centre for various critical diseases. It is largest and most sophisticated government hospital of Himachal.
There are two NICU \& one pediatrics ICU under the department of Pediatrics. Head of department and senior professor is in-charge of NICU. Junior residents and nursing staff are responsible for full time service delivery in NICUs. Junior residents generally have a posting of one month on rotation basis. Presently 15 staff nurses and 20 residents (Junior residents \& Senior residents). Thus, sample size of 35 was covered. Those HCP not providing consent \&Junior residents having a posting of less than one month were excluded. The study consist of the assessment of knowledge and practices among study participants regarding standard precautions and other essential information on standard infection control protocols. This was done using a predesigned, pretested semi structured interview schedule after taking written informed consent from the study subjects. Data were collected, compiled and entered into MS excel spread sheet and statistical analysis was done using SPSS Version 20 and appropriate statistical tests were applied.

\section{Results}

The knowledge and awareness of HCAI in ICU staff. Total ICU staff interviewed was 35 out of which 15 were doctors and rest nursing staff. Socio-demographic profile as shown in table 1 . Out of 15 doctors 3 were SRs, while 12 were JRs. Out of 20 Nursing staff 2 were GNM qualified while 18 were B.Sc. Nursing qualified. Doctors were having an average experience of 5 years while nursing staff were having an average experience of 15.9 years of working in healthcare. ICU experience of doctors was also less as compare. Knowledge and awareness regarding various parameters are shown in table 2.

Table1: Socio demographic profile

\begin{tabular}{|l|l|c|c|c|}
\hline \multicolumn{2}{|l|}{ Variables } & Doctor & Nursing staff & Total \\
\hline \multicolumn{2}{|l|}{ Mean age (yrs) } & 32.6 & 39.7 & 36.8 \\
\hline \multirow{2}{*}{ Sex } & Male & 7 & 2 & 9 \\
\cline { 2 - 5 } & Female & 8 & 18 & 26 \\
\hline \multirow{2}{*}{$\begin{array}{l}\text { Marital } \\
\text { status }\end{array}$} & Married & 5 & 19 & 24 \\
\cline { 2 - 5 } & Unmarried & 10 & 1 & 11 \\
\hline \multicolumn{2}{|l|}{ Total } & 15 & 20 & 35 \\
\hline
\end{tabular}


Table 2: Knowledge and awareness about various variables for hospital infection control

\begin{tabular}{|c|c|c|c|c|}
\hline \multicolumn{2}{|l|}{ Knowledge about } & Doctor & Nursing staff & Total \\
\hline \multirow[t]{2}{*}{ Definition of HCAI } & Present & 12 & 12 & 24 \\
\hline & Absent & 3 & 8 & 11 \\
\hline \multirow[t]{2}{*}{ HCAI as a major health problem } & Present & 15 & 20 & 35 \\
\hline & Absent & 0 & 0 & 0 \\
\hline \multirow[t]{2}{*}{ Infection Control Committee } & Present & 2 & 2 & 4 \\
\hline & Absent & 13 & 18 & 31 \\
\hline \multirow[t]{2}{*}{ Infection control guidelines } & Present & 6 & 10 & 16 \\
\hline & Absent & 9 & 10 & 19 \\
\hline \multirow[t]{2}{*}{ Infection control policy } & Present & 1 & 19 & 20 \\
\hline & Absent & 14 & 1 & 15 \\
\hline \multirow{2}{*}{$\begin{array}{lllll}\begin{array}{l}\text { Knowledge } \\
\text { hygiene }\end{array} & \& \text { awareness } & \text { about hand } \\
\end{array}$} & Present & 15 & 20 & 35 \\
\hline & Absent & 0 & 0 & 0 \\
\hline \multirow[t]{2}{*}{ Knowledge about hand washing set up } & Present & 14 & 15 & 29 \\
\hline & Absent & 1 & 5 & 6 \\
\hline \multirow{2}{*}{ Practice of hand hygiene } & Always & 15 & 18 & 33 \\
\hline & Not always & 0 & 2 & 2 \\
\hline \multirow[t]{2}{*}{ Knowledge of PPE } & Present & 14 & 14 & 28 \\
\hline & Absent & 1 & 6 & 7 \\
\hline \multirow[t]{2}{*}{ Practice of personal protection } & Always & 12 & 16 & 8 \\
\hline & Not always & 3 & 4 & 7 \\
\hline \multirow[t]{2}{*}{ Knowledge of HIV \& HbsAg transmission } & Present & 15 & 16 & 31 \\
\hline & Absent & 0 & 4 & 4 \\
\hline \multirow[t]{2}{*}{ Hep-B vaccination } & Done & 14 & 20 & 34 \\
\hline & Not done & 1 & 0 & 1 \\
\hline \multirow[t]{2}{*}{ Universal protection practice } & Always & 14 & 14 & 28 \\
\hline & Not always & 1 & 6 & 7 \\
\hline \multirow[t]{2}{*}{ Knowledge of BMW management } & Present & 15 & 20 & 35 \\
\hline & Absent & 0 & 0 & 0 \\
\hline \multirow[t]{2}{*}{ Knowledge of protection from swine flu } & Present & 13 & 18 & 31 \\
\hline & Absent & 2 & 2 & 4 \\
\hline
\end{tabular}

\section{Discussion}

Total of 35 ICU staff were interviewed, working in that particular month of assessment. Findings suggests that all the staff were qualified enough as per their designation. And approx. 50\% of the staff were having adequate experience of ICU. Doctors were more aware about definition HCAI as compared to nursing staff. And most of the ICU staff considered HCAI as a major problem in healthcare. Kotwal et al also reported similar findings in his study conducted at a tertiary care center. $^{3}$

The present study reports that nursing staff more aware of Infection control committee in comparision to doctors. This may be explained by the fact that 12 out of 15 doctors were post graduate student who were not having much experience of working in a hospital specially ICU setup. On comparing knowledge of infection control guidelines doctors were found to be more aware than nursing staff, as $50 \%$ of the nursing staff were found to be unaware about it. This is in contrast to findings reported by Alrubaiee et al. ${ }^{4}$

The present study reports that approx. $76 \%$ of the staff was aware of hand hygiene. However approx. $50 \%$ of the staff was not practicing it regularly. Similar findings were also reported by Taneja et al. In contrast Anupriya et al reported that hand hygiene was top most priority of ICU staff. The reason for not following hand hygiene regulary was work load. ${ }^{5,6}$. Approx. $24 \%$ of the staff was not knowing about the component of proper hand washing set up. Majority of the staff pointed lack of hand dryer and Majority of the staff were knowing about and were doing personal protective equipment practices. Although in comparison to doctors, nursing staff were less aware and less frequently practicing it. Similar finding were also reported by Kotwal et al disposable towels in the ICU. ${ }^{3}$ 
In comparison to nursing staff doctors were more aware about HIV and HbsAg transmission, more than $50 \%$ of nursing staff were unaware about it. All of the staff were vaccinated for Hep-B except 1 doctor. Majority of the staff was knowing about protective measures of swine flu. Although approx. $24 \%$ of the staff was unaware about it. Majority of the staff was following universal protection practice while dealing with HIV and HbsAg +ve patients. This is in contrast to previous study by Kotwal et al, in which he reported that a significant proportion of staff was not following universal precautions. ${ }^{3}$

In comparison to nursing staff, doctors were found to be more aware about BMW management. In contrast Anupriya et al reported good BMW management practices among nursing staff. ${ }^{6}$

\section{Conclusion}

Infection control committee of a hospital plays a vital role in controlling HCAI by making infection control policy and guidelines. But at the same time awareness should also be there among health care providers especially in Intensive care units, where in patients with severe infections are frequently admitted. This awareness can only be achieved through proper training at frequent interval. Central sterile and supply department should be made according to standard guidelines to prevention HCAI. Linen and laundry services should ideally be within premises of a large hospital and proper wash formula should be used. Adequate staff is also important, otherwise because of workload staff would not follow the proper washing instructions. Staffing of ICUs should also be adequate according to standard laid down by WHO which is unfortunately lacking in most places especially in developing countries like India. Hand hygiene is of most important in preventing HCAI. But, unfortunately it is neglected in Indian setting specially in government setup where work load is in excess and lack of materials required for hand hygiene are lacking. Finally staff should be motivated to follow infection control guidelines, hand hygiene, personal protection, and trained enough, so that, this major problems of HCAI can be controlled for the benefit of patients and health care providers.

\section{References}

1. World Health Organization. Prevention of hospital acquired infections [Internet]. Geneva: WHO;2002. Available from: http//www.who.int.

2. World Health Organization. Report on the burden of endemic health care associated infections worldwide. Geneva: WHO; 2011. Available from: http//www.who.int.

3. Kotwal A, Taneja DK. Health careworkers and universal precautions: perceptions and determinants of non-compliance. Indian $\mathbf{J}$ of Community Medicine. 2010;35(4): 268.

4. Alrubaiee et al. Safety in Health (2017) 3:16

5. Taneja J, BhibhaBati M, Aradhana B, Poonam L, Vinita D, Archana T. evaluation of knowledge and practices amongst nursing staff toward infection control measures in a tertiary care hospital in India. Can J Infect Control.2009;24(2): 104-7.

6. Anupriya A, Priyanka N, Snehalaxmi R, Uma A. Health-care associated infections and infection control practices in intensive care unit of a tertiary care Hospital. Asian J Pharm Clin Res. 2016;(9)4: 399-402. 